\title{
Chief Physician Wang Defen's Experience in Treating Thyroid Nodules based on Syndrome Differentiation
}

\author{
Tian Zhao', Defen Wang2*, Mengyu Zhang1, Jinjin Chai1 \\ ${ }^{1}$ Shaanxi University of Chinese Medicine, Xianyang 712046, Shaanxi Province, China \\ ${ }^{2}$ Xi'an Hospital of Traditional Chinese Medicine, Xi'an 710021, Shaanxi Province, China \\ *Corresponding author: Defen Wang, wangdefen69@sohu.com
}

\begin{abstract}
To summarize the experience of Chief Physician Wang Defen in the treatment of thyroid nodules. According to many years of clinical experience, Chief Physician Wang Defen believes that the occurrence of thyroid nodules is mostly affected by emotional factors. Qi stagnation, liver depression and fire, phlegm and Qi are the pathogenesis of the disease, which is closely related to liver Qi stagnation. Based on the basic treatment principle of Nourishing Yin, clearing heat, soothing liver and strengthening spleen, the prescription chose Danzhi Xiaoyao Powder plus or minus can often achieve good clinical efficacy.
\end{abstract}

Keywords: Thyroid nodules; Danzhi Xiaoyao Powder; Syndrome differentiation and treatment; Experience

Publication date: November 2021; Online publication: November 30, 2021

\section{Introduction}

Thyroid nodules are masses with one or more abnormal tissue structures in the thyroid due to various factors ${ }^{[1]}$. The incidence rate of thyroid nodules is increasing, but most of them are benign nodules. $5 \%$ to $15 \%$ of thyroid nodules are thyroid cancer ${ }^{[2]}$. Most patients with thyroid nodules have no clinical symptoms, and some may have hoarseness, swallowing discomfort, dyspnea and so on. The author studied with Chief Physician Wang Defen and the clinical application of traditional Chinese medicine in the treatment of thyroid nodules showed unique advantages.

\section{Etiology and pathogenesis}

Modern medicine shows that the main pathogenic factors are iodine deficiency, autoimmunity and heredity. In terms of treatment, levothyroxine, radioactive iodine, absolute ethanol injection and surgical treatment are mainly used ${ }^{[3]}$. Thyroid diseases belong to the category of "goiter" in traditional Chinese medicine. Professor Xianchun $\mathrm{Bu}^{[4]}$ believes that the pathogenesis of this disease is related to the liver. According to "Lingshu - Channel Vessels": "The Jueyin vein of the liver foot. The branches are in the flank and the straight branches run up the back of the throat. Xiaodan Jin ${ }^{[5]}$ believes that the basic pathogenesis is the loss of liver drainage and Qi stagnation, followed by pathological factors such as Qi, fire, phlegm and blood stasis. If it is blocked in front of the neck, it is a nodule. Bo $\mathrm{Li}^{[6]}$ believes that if the mood is not adjusted, the liver Qi is stagnant, so that the Qi of the whole body is lost in catharsis, the blood and body fluid cannot be transmitted normally, the phlegm is stagnant in the neck, and goiter and tumor are formed after a long time. Lan Zhang ${ }^{[7]}$ believed that the disease was mainly caused by the loss of liver and the deficiency of spleen and kidney. In conclusion, the production of thyroid nodules is related to the drainage of liver. 


\section{Pattern identification as the basis for determining treatment}

Chief Physician Wang Defen believes that the pathogenesis of thyroid nodules is closely related to liver depression, and divides them into Qi depression and phlegm knot syndrome, liver fire exuberance syndrome, turbid phlegm and blood stasis syndrome and Qi and Yin deficiency syndrome for syndrome differentiation and treatment. Xiaoyao Powder is derived from the prescription of Taiping Huimin Hejiju Perscription. It is mainly used to treat the syndrome of liver depression, spleen weakness and blood deficiency. If the liver is depressed and turns into fire, the internal and external heat is abundant, and peony skin and gardenia are added. This is Jiawei Xiaoyao Powder, also known as Danzhi Xiaoyao Powder ${ }^{[8]}$ Radix Bupleuri in the prescription can soothe the liver, relieve depression and regulate Qi; Radix Angelicae Sinensis nourishes and promotes blood circulation; Radix Paeoniae Alba softens the liver, nourishes Yin, replenishes blood and harmonize blood; Seeing the disease of the liver, we know that the liver transmits the spleen, so we use Rhizoma Atractylodis Macrocephalae, Poria cocos and Radix Glycyrrhizae to warm and strengthen the spleen, replenish Qi and replenish the middle-Jiao; Fructus Gardeniae purges heart fire, and clears the fire of triple energizers; Peony bark clears heat and sooths liver; Peppermint soothes the liver and dissipates heat. The whole prescription makes the liver Qi regulation, spleen health transport, Qi and blood regulation, heat removal.

\section{Medical records}

Ms. Wang, female, 46 years old, was initially diagnosed on June 11, 2021 because "thyroid nodules were found for more than 2 years." The patient reported that a hypoechoic nodule of about $5.8 \mathrm{~mm} \times 7.0 \mathrm{~mm}$ was found in the right lobe of the thyroid gland by thyroid ultrasound during the physical examination two years ago. It has clear boundary, medium texture, clear boundary with surrounding tissues, and uneven parenchymal echo; Thyroid function is normal. Thus, she came to the outpatient department of our hospital. Initial diagnosis was: irritability, a feeling of distension in front of the neck, dry throat, sore throat, fatigue, knee pain, no chest tightness, shortness of breath, no swallowing obstruction, the patient complained of high work pressure, can sleep, and normal stool and urine. The tongue was red, the coating was white and dry, and the number of pulse strings was high. TCM diagnosis was: goiter and tumor (liver depression, spleen deficiency and blood stasis). Western medicine diagnosis was: thyroid nodule.

Treatment was: soothe the liver and strengthen the spleen, remove blood stasis and disperse nodule. Prescription was: addition and subtraction of Danzhi Xiaoyao Powder. The details of prescription are as follows: Paeonia suffruticosa 15g, Fructus Gardeniae Praeparatus 6g, Radix Angelicae Sinensis 15g, Radix Paeoniae Alba 30g, bran fried atractylodes 15g, Poria cocos 15g, vinegar Radix Bupleuri $6 \mathrm{~g}$, roasted Radix Glycyrrhizae 6g, wine Radix Scutellariae 6g, Rhizome Pinelliae Preparata 10g, stir-fried toosendan 10g, Rhizoma Ligustici 10g, vinegar Faeces Togopteri 15g, bran-fried bitter orange 12g, and Herba Eupatorii 15g. It totally was 6 dose, fried in water. Nursing was: to regulate mood, eat less iodine and exercise appropriately. Second diagnosis: The patient complained that the above symptoms were relieved, the tongue was red, the coating was thin and white, and the number of pulse strings was high. The prescription remains the same and continues to take. Third diagnosis: The patient complained obvious improvement of irritability and the feeling of suffocation in front of the neck, no obvious other discomfort, red tongue, thin white coating and thin pulse string. A hypoechoic nodule of about $3.8 \mathrm{~mm} \times 4.6 \mathrm{~mm}$ was found in the right lobe of the thyroid gland by thyroid ultrasound. It has clear boundary. Thyroid function is normal. $20 \mathrm{~g}$ of Radix Scrophulariae, 15g of Radix Rehmanniae Preparata and 15g of Salvia Beverage was added to the prescription above, and the prescription was decocted in water for oral dose. It is recorded as the patient is a middle-aged woman with high work pressure, emotional disorder, poor liver meridian and functioning of Qi, so her chest is stuffy, liver depression turns into fire and disturbs her heart, so she is upset and irritable; Phlegm and blood stasis are combined in the pharynx, so there is a feeling of neck suffocation; Qi stagnation 
turns into fire, hurts the body fluid and causes dry throat and sore throat; Liver Qi travels by the spleen, and the spleen and stomach are weak, so the spirit is tired and weak. Combined with the syndrome differentiation of tongue and pulse, it is the syndrome of liver depression, spleen deficiency and blood stasis. Danzhi Xiaoyao Powder is added and subtracted to soothe the liver, strengthen the spleen, activate blood and remove blood stasis.

\section{Summary}

The liver likes regulate, invigorate and hates depression, which plays an important role in the smooth discharge of human Qi and the pivotal transport of Qi, blood and body fluid. Chief Physician Wang Defen combined with her many years of clinical experience, often used Danzhi Xiaoyao Powder to treat thyroid nodules, addressing both symptoms and root causes, with remarkable curative effect.

\section{Disclosure statement}

The authors declare no conflict of interest.

\section{References}

[1] Teng W, Zeng Z, Li G, et al., 2008, Guide for Diagnosis and Treatment of Thyroid Diseases in China. Chinese Society of Endocrinology, 6-60.

[2] Mehanna H, Jain A, Morton RP, et al., 2009, Investigating the Thyroid Nodule. Clinical Otolaryngology, 338(7696): 705-709.

[3] Li B, Liu Q, Ding X, et al., 2017, Progress in Modern Diagnosis and Treatment of Thyroid Nodules, Advances in Modern Biomedicine, 17(19): 7.

[4] Yang Z, Qiu S, Xiao L, et al., 2018, Bu Xianchun's Experience in Treating Thyroid Nodules with Chaihu Shugan Powder. Hunan Journal of Traditional Chinese Medicine. 34(10): 2.

[5] Jin X, Yu J, 2021, The Pathogenesis and Prevention of Thyroid Nodules based on the Theory of "Six Yu." Chinese Journal of Traditional Chinese Medicine, 36(5): 3.

[6] Qin S, Kang X, 2018, Summary of Professor Kang Xuedong's Experience in the Treatment of Benign Thyroid Nodules by Soothing the Liver and Strengthening the Spleen. Asia-Pacific Traditional Medicine. 14(12): 2.

[7] Li B, Li C, 2020, Effect of Xiaoyao Powder Addition and Subtraction Prescription in the Treatment of Benign Thyroid Nodules and Case Study. Journal of Contemporary Medicine, 18(2): 3.

[8] Zhu C, Zhang L, 2021, Zhang Lan's Experience in the Treatment of Thyroid Nodules. Shanxi Traditional Chinese Medicine, 37(5): 11-12. 